\title{
Pressure dependence of the superconducting and magnetic transition temperatures in $\mathrm{Sr}_{2} \mathrm{VO}_{3} \mathrm{FeAs}$
}

\author{
S. Holenstein $\odot,{ }^{1,2,{ }^{*}}$ F. Hummel, ${ }^{3}$ Z. Guguchia $\odot,{ }^{1}$ S. Kamusella, ${ }^{4}$ N. Barbero, ${ }^{5}$ H. Ogino, ${ }^{6}$ Z. Shermadini,${ }^{1,7}$ R. Khasanov, ${ }^{1}$ \\ A. Amato, ${ }^{1}$ T. Shiroka $\odot,{ }^{1,5}$ H.-H. Klauss, ${ }^{4}$ E. Morenzoni, ${ }^{1,2}$ D. Johrendt $\odot,{ }^{3}$ and H. Luetkens $\oplus^{1, \dagger}$ \\ ${ }^{1}$ Laboratory for Muon Spin Spectroscopy, Paul Scherrer Institute, CH-5232 Villigen PSI, Switzerland \\ ${ }^{2}$ Physik-Institut der Universität Zürich, Winterthurerstrasse 190, CH-8057 Zürich, Switzerland \\ ${ }^{3}$ Department Chemie, Ludwig-Maximilians-Universität München, Butenandtstrasse 5-13 (D), 81377 München, Germany \\ ${ }^{4}$ Institute of Solid State and Materials Physics, TU Dresden, DE-01069 Dresden, Germany \\ ${ }^{5}$ Laboratorium für Festkörperphysik, ETH Zürich, CH-8093 Zurich, Switzerland \\ ${ }^{6}$ National Institute of Advanced Industrial Science and Technology (AIST), Tsukuba 305-8568, Japan \\ ${ }^{7}$ I. Javakhishvili Tbilisi State University, 0179 Tbilisi, Georgia
}

(Received 13 September 2019; revised 12 August 2021; accepted 18 August 2021; published 16 September 2021)

\begin{abstract}
We report muon spin rotation $(\mu \mathrm{SR})$ and magnetization measurements on superconducting $\mathrm{Sr}_{2} \mathrm{VO}_{3} \mathrm{FeAs}$ under pressure. At ambient pressure, $\mathrm{Sr}_{2} \mathrm{VO}_{3} \mathrm{Fe}$ As undergoes an antiferromagnetic transition of the $\mathrm{V}$ moments at $T_{N}$ and becomes superconducting at $T_{c}<T_{N}$. As a function of pressure, $T_{N}$ initially decreases while $T_{c}$ increases. Surprisingly, once $T_{N} \approx T_{c}$ at $0.6 \mathrm{GPa}, T_{N}$ reverses its trend and increases together with $T_{c}$, which might indicate a cooperative coupling of the superconducting and the magnetic order.
\end{abstract}

DOI: 10.1103/PhysRevB.104.104507

\section{INTRODUCTION}

Superconductivity and magnetism are normally considered to be antagonistic. Nonetheless, there are several examples of long-range magnetic order coexisting with superconducting order. In cases with a large spatial separation of the localized orbitals of the atom responsible for the magnetism and the superconducting electron system, there is no or only a weak coupling between the two orders. Examples are the Chevrel phases $\left(R \mathrm{Mo}_{6} \mathrm{~S}_{8}\right.$, where $R$ denotes rare earth) $[1,2]$, the borocarbides $R \mathrm{Ni}_{2} \mathrm{~B}_{2} \mathrm{C}[3,4]$, the ruthenate $\mathrm{RuSr}_{2} \mathrm{GdCu}_{2} \mathrm{O}_{8}[5,6]$, and the iron-based superconductor $\mathrm{EuFe}_{2} \mathrm{As}_{2}$ doped either with $\mathrm{P}$ or $\mathrm{Ru}[7,8]$. Sizable coupling has been observed for the ferromagnetic order below $1 \mathrm{~K}$ in $\mathrm{UGe}_{2}$ and URhGe [9-11]. A coupling of antiferromagnetic [spin density wave (SDW)] and superconducting order can be observed at much higher temperatures, e.g., in iron-based superconductors [12-23]. In the case of a microscopic coexistence, the coupling is normally found to be of a competitive nature [16-23]. In FeSe, the coupling changes from competitive at lower pressures to a cooperative behavior at higher pressures [24,25]. Finding and understanding different forms of coexistence and coupling between magnetic and superconducting orders is not only relevant for the search for higher superconducting transition temperatures $T_{c}$, but it might also be interesting for technical applications if one order can be manipulated by controlling the other.

The iron-based superconductor $\mathrm{Sr}_{2} \mathrm{VO}_{3} \mathrm{Fe}$ As exhibits superconductivity below $T_{c} \approx 37 \mathrm{~K}$ at ambient pressure [26] and $46 \mathrm{~K}$ at $p=4 \mathrm{GPa}$ [27]. $\mathrm{Sr}_{2} \mathrm{VO}_{3} \mathrm{FeAs}$ is composed of alternating conducting $\mathrm{FeAs}$ and $\mathrm{Sr}_{2} \mathrm{VO}_{3}$ buffer layers [26]. Upon

\footnotetext{
*stefan.holenstein@psi.ch

${ }^{\dagger}$ hubertus.luetkens@psi.ch
}

lowering the temperature, $\mathrm{Sr}_{2} \mathrm{VO}_{3} \mathrm{Fe}$ As undergoes an antiferromagnetic transition presumably of the $\mathrm{V} 3 d$ moments before it becomes superconducting with the FeAs layer being nonmagnetic $[28,29]$. Since the V $3 d$ state is less localized than, e.g., the $\mathrm{Gd} 4 f$ state in $\mathrm{RuSr}_{2} \mathrm{GdCu}_{2} \mathrm{O}_{8}[5,6]$ and hybridizes with the $\mathrm{Fe} 3 d$ state [30,31], $\mathrm{Sr}_{2} \mathrm{VO}_{3} \mathrm{Fe} A$ s has the potential to exhibit significant coupling between the superconducting and the magnetic order.

In this work, we present a study of the superconducting and magnetic order in $\mathrm{Sr}_{2} \mathrm{VO}_{3} \mathrm{FeAs}$ under hydrostatic pressure up to $2.2 \mathrm{GPa}$ by means of muon spin rotation and relaxation $(\mu \mathrm{SR})$ and dc-magnetization measurements. We find that the magnetic ordering temperature $T_{N}$ initially decreases with pressure, while the superconducting transition temperature $T_{c}$ increases. At a pressure of $p \approx 0.6 \mathrm{GPa}$, the two ordering temperatures become comparable. Surprisingly, at higher pressures, $T_{N}$ increases again together with $T_{c}$, with the superconductivity setting in shortly below the magnetic order. We discuss possible explanations for this peculiar phase diagram.

\section{SAMPLE SYNTHESIS AND EXPERIMENTAL METHODS}

Polycrystalline $\mathrm{Sr}_{2} \mathrm{VO}_{3} \mathrm{FeAs}$ was synthesized and characterized following Ref. [29]. The sample contains 3.2\% $\mathrm{Sr}_{3} \mathrm{~V}_{2} \mathrm{O}_{7-x}, 2.2 \%$ orthorhombic $\mathrm{Sr}_{2} \mathrm{VO}_{4}$, and $3.3 \% \mathrm{FeAs}$, but it does not exhibit oxygen deficiency or $\mathrm{V}$ at the iron site. The superconducting transition temperature is $T_{c} \approx 25 \mathrm{~K}$ and the diamagnetic shielding fraction is about $26 \%$. These values are comparably low, but similar values have been reported before $[32,33]$. The actual superconducting volume fraction of our sample is undetermined since the relation to the diamagnetic shielding fraction is nontrivial for a polycrystalline sample with small grains. $\mu \mathrm{SR}$ measurements were performed at the Swiss Muon Source $\mathrm{S} \mu \mathrm{S}$ using the GPS [34] 


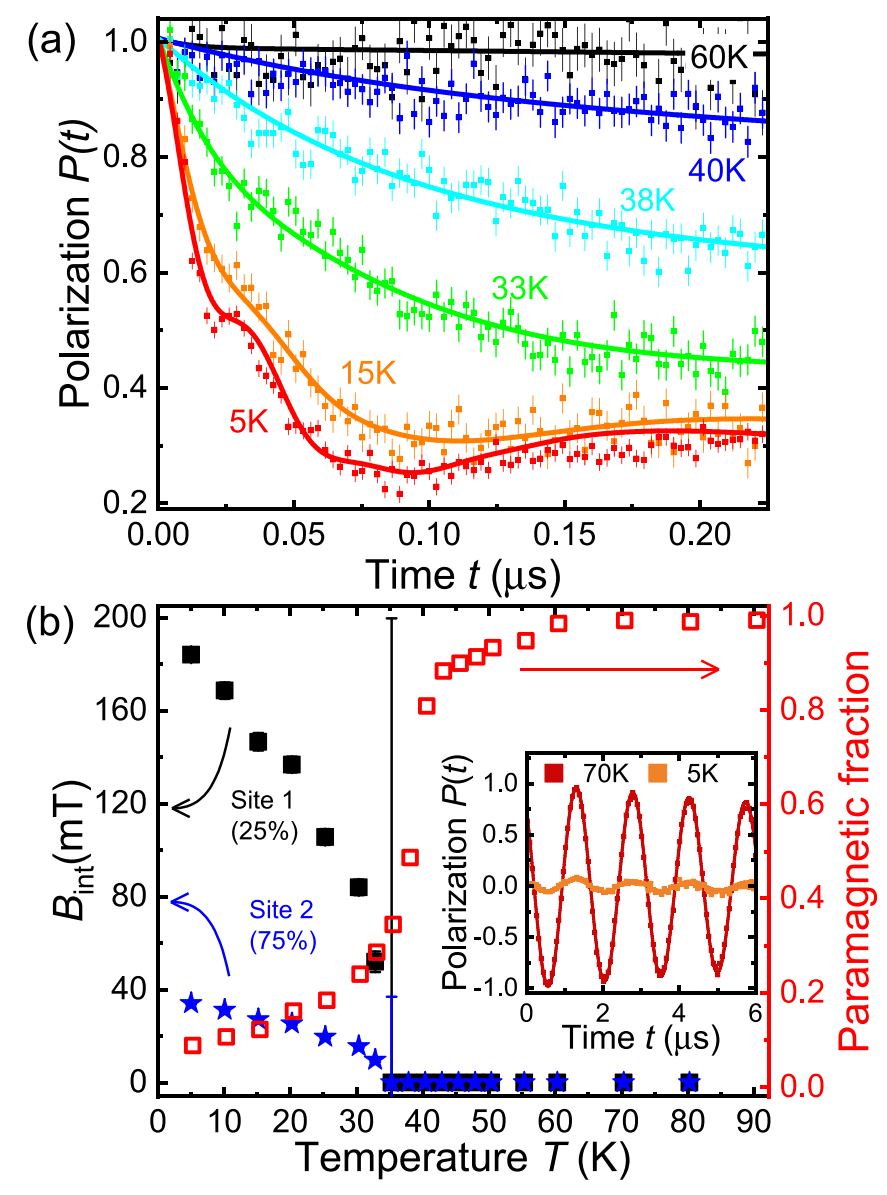

FIG. 1. (a) Representative zero-field (ZF) $\mu$ SR spectra of polycrystalline $\mathrm{Sr}_{2} \mathrm{VO}_{3} \mathrm{FeAs}$ at ambient pressure. The (heavily damped) oscillations at lower temperatures are due to the onset of static, long-range magnetic order. The solid lines are fits using the model introduced in Eq. (1). (b) Left axis: Internal magnetic field $B_{\text {int }}$ at the minority (black squares) and majority (blue stars) muon stopping sites as a function of temperature. At $35 \mathrm{~K}$, the determination of the small $B_{\text {int }}$ is difficult due to strong damping of the signal, leading to very large error bars. At the other temperatures, the error bars are smaller than the data point symbols. Right axis: Paramagnetic fraction (red open squares) of the $\mathrm{Sr}_{2} \mathrm{VO}_{3} \mathrm{FeAs}$ sample as a function of temperature determined by $5 \mathrm{mT}$ transverse-field (TF) $\mu \mathrm{SR}$. Inset: Representative $5 \mathrm{mT}$ TF $\mu$ SR spectra. The paramagnetic fraction of the sample is determined from the oscillation amplitude.

and GPD [35] spectrometers. The data were analyzed with the free software package MUSRFIT [36]. dc-magnetization measurements were performed using a commercial superconducting quantum interference device (SQUID) magnetometer. Hydrostatic pressure for the $\mu \mathrm{SR}$ measurements was applied using a double-wall piston cell [35]. A CuBe anvil-type cell with diamond anvils was used for the dc-magnetization measurements. Pressures were determined by either In or $\mathrm{Pb}$ manometers [37], and Daphne 7373 oil was used as a pressure-transmitting medium.

\section{III. $\mu$ SR AND MAGNETIZATION MEASUREMENTS}

Figure 1(a) shows representative zero-field ( $\mathrm{ZF}$ ) muon spin polarization spectra $P(t)$. Down to $60 \mathrm{~K}$, no sign of magnetism is observed, ruling out a magnetic transition claimed previously in the $150-170 \mathrm{~K}$ temperature region [32,38]. Below $60 \mathrm{~K}$, the relaxation rate increases and spontaneous muon spin-precession with two distinct frequencies can be observed below $35 \mathrm{~K}$, indicating the onset of static, long-range magnetic order. The spectra were fitted by the sum of a paramagnetic $\left[P_{\mathrm{pm}}(t)\right]$ and a magnetic $\left[P_{\mathrm{magn}}(t)\right]$ contribution, assuming that the two distinct precession frequencies are due to two different muon stopping sites within the crystal lattice:

$$
P_{\text {sample }}(t)=f_{m} P_{\text {magn }}(t)+\left(1-f_{m}\right) P_{\mathrm{pm}}(t),
$$

where

$$
\begin{gathered}
P_{\text {magn }}(t)=f_{1}\left[\frac{2}{3} \cos \left(\gamma_{\mu} B_{\mathrm{int}, 1} t\right) e^{-\lambda_{\mathrm{T}, 1} t}+\frac{1}{3} e^{-\lambda_{\mathrm{L}, 1} t}\right] \\
+\left(1-f_{1}\right)\left[\frac{2}{3} \cos \left(\gamma_{\mu} B_{\mathrm{int}, 2} t\right) e^{-\lambda_{\mathrm{T}, 2} t}+\frac{1}{3} e^{-\lambda_{\mathrm{L}, 2} t}\right] \\
P_{\mathrm{pm}}(t)=\operatorname{GKT}(t) e^{-\lambda_{\mathrm{pm}} t}
\end{gathered}
$$

Here, $f_{m}$ is the magnetic volume fraction, $f_{1}$ is the fraction of muons stopping at site $1, \gamma_{\mu}=2 \pi \times 135.5 \mathrm{MHz} / \mathrm{T}$ is the muon's gyromagnetic ratio, $B_{\text {int, } \mathrm{i}}$ is the magnetic field at the respective muon site, and $\lambda_{\mathrm{T}, \mathrm{i}}$ and $\lambda_{\mathrm{L}, \mathrm{i}}$ are the so-called transverse and longitudinal relaxation rates for the respective muon site. The 2/3 (transverse) and 1/3 (longitudinal) components reflect the polycrystalline nature of the sample leading to a powder average of the internal fields with respect to the initial muon spin direction. The paramagnetic fraction is modeled by the product of a static Gaussian Kubo-Toyabe function [39] and an exponential relaxation with relaxation rate $\lambda_{\mathrm{pm}}$. To make the fit more stable and to reflect the fact that the muons at both stopping sites observe the same magnetic structure, just from different positions within the unit cell, the parameters $B_{\text {int, } 1}$ and $B_{\text {int,2 }}$ as well as $\lambda_{\mathrm{L}, 1}$ and $\lambda_{\mathrm{L}, 2}$ were coupled with a proportionality constant.

The analysis yields $f_{1}=0.25$ and $B_{\text {int }, 1} / B_{\text {int }, 2}=5.4$. Figure 1(b) shows the temperature dependence of the internal fields $B_{\text {int }}$ at the muon stopping sites $1(25 \%)$ and $2(75 \%)$. Also shown in Fig. 1(b) is the paramagnetic fraction $\left(1-f_{m}\right)$ of the sample determined from the oscillation amplitude of the weak transverse-field (TF) $\mu$ SR spectra [cf. the inset of Fig. 1(b) for representative spectra]. These data show that $\mathrm{Sr}_{2} \mathrm{VO}_{3} \mathrm{FeAs}$ exhibits static, long-range magnetic order with a nearly full volume fraction that microscopically coexists with the superconducting volume of our sample. The internal field at site 2 is comparable to the single field reported in an earlier study on oxygen-deficient $\mathrm{Sr}_{2} \mathrm{VO}_{3} \mathrm{FeAs}$ [28]. The significantly larger internal field we detect at site 1 was not reported previously. Possibly, it was overlooked due to the small signal fraction ( $25 \%$ ) and the relatively strong damping. It is worth noting that there is neither a reduction of $B_{\text {int }}$ (which is proportional to the ordered magnetic moment) nor a reduction of the magnetic volume fraction below $T_{c} \approx 25 \mathrm{~K}$. A reduction would be expected in the case of competition between the magnetic and superconducting order parameter or volume [24,25].

${ }^{57} \mathrm{Fe}$ Moessbauer measurements in earlier studies [28,32] as well as on our sample (Appendix C) show that the $\mathrm{Fe}$ moments do not participate in the static magnetic order and 


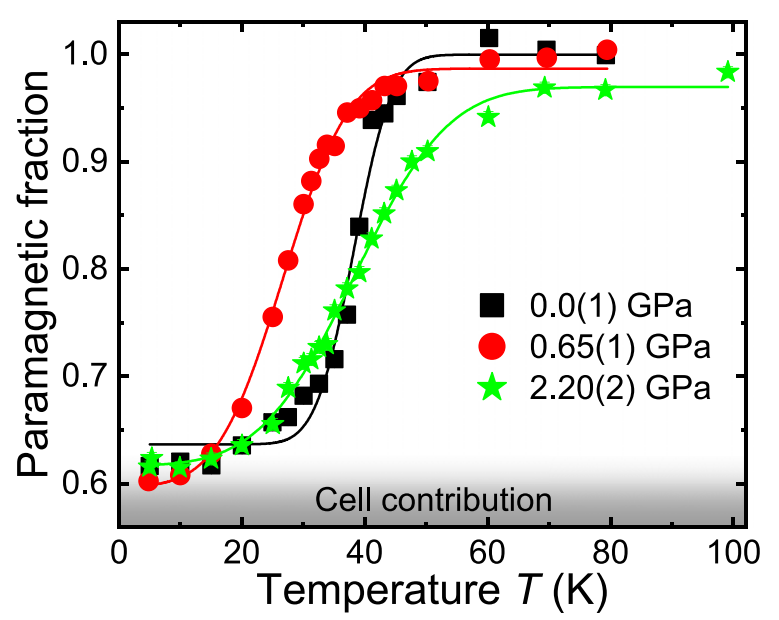

FIG. 2. Paramagnetic fraction of $\mathrm{Sr}_{2} \mathrm{VO}_{3} \mathrm{FeAs}$ as a function of temperature for representative pressure points determined by $\mathrm{TF}$ $\mu \mathrm{SR}$ at $5 \mathrm{mT}$. The error bars are smaller than the data point symbols. The sample is virtually fully magnetic at low temperatures for all pressures. The remaining $60 \%$ of the paramagnetic signal is due to muons stopping in the pressure cell. The solid lines are fits using a normal cumulative distribution function assuming a Gaussian distribution of magnetic transition temperatures.

therefore imply V ordering. Reports on ${ }^{51} \mathrm{~V}$ and ${ }^{75}$ As nuclear magnetic resonance (NMR) are inconsistent since they either claim Fe magnetism [40,41] or argue in favor of V magnetism [27,38]. NMR measurements on our sample (Appendix D) are in agreement with the literature data to a large extent, but they cannot provide a conclusive answer as to which element carries the ordering moments. Also, it is not known whether the high magnetic fields required for NMR influence the magnetic properties in a significant way. Since ${ }^{57} \mathrm{Fe}$ Moessbauer spectroscopy, like $\mu \mathrm{SR}$, is performed under (nearly) zero field conditions, we are confident that it is the $\mathrm{V}$ that magnetically orders in $\mathrm{Sr}_{2} \mathrm{VO}_{3} \mathrm{FeAs}$.

Motivated by the large and positive pressure effect on the superconducting transition temperature [27,42], we performed $\mu \mathrm{SR}$ measurements under hydrostatic pressures up to $2.2 \mathrm{GPa}$. Figure 2 shows the paramagnetic fraction as a function of temperature determined by weak TF $\mu$ SR for representative pressures. Please note that $60 \%$ of the muons stop in the pressure cell, meaning that for all pressures the magnetic fraction of the sample stays close to $100 \%$ at low temperatures. Assuming a Gaussian distribution of magnetic transition temperatures, the temperature dependence of the paramagnetic fraction was modeled by a normal cumulative distribution function (solid lines in Fig. 2). Figure 3 shows the magnetic transition temperature $T_{N}$ determined from the midpoint of these curves. The error bars represent the uncertainty of the fit parameter. At ambient pressure, $T_{N}$ determined by this method coincides reasonably well with the onset of spontaneous muon spin precession observed by ZF $\mu$ SR.

In addition, we performed measurements of the superconducting transition temperature by means of SQUID magnetometry under pressure (Appendix B). The obtained onset temperatures for superconductivity are depicted in Fig. 3. In agreement with the literature data, $T_{c}$ increases monoton-

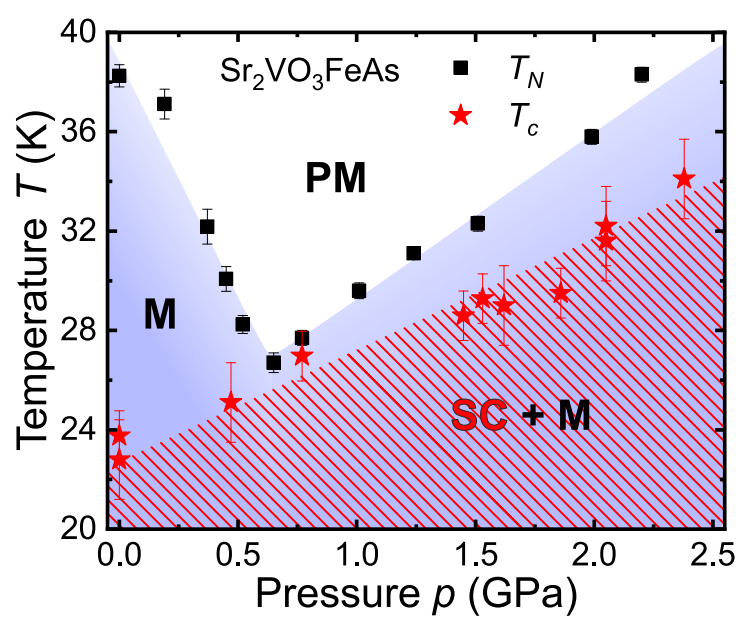

FIG. 3. Temperature-pressure phase diagram of $\mathrm{Sr}_{2} \mathrm{VO}_{3} \mathrm{FeAs}$. The magnetic transition temperatures $T_{N}$ (black squares) were determined from the midpoint of the temperature dependence of the paramagnetic fraction (Fig. 2). The onset superconducting transition temperatures $T_{c}$ (red stars) were measured by dc-magnetization measurements. Below 0.6 GPa, the static, long-range magnetic $(M)$ order is suppressed with increasing pressure while the superconducting order (SC) is enhanced until $T_{c} \approx T_{N}$. Above $0.6 \mathrm{GPa}$, the trend of $T_{N}$ is reversed and $T_{N}$ and $T_{c}$ increase simultaneously.

ically with pressure [27,42]. The superconducting volume fraction stays roughly constant for all measured pressures. The magnetic transition temperature initially decreases with increasing pressure, until $T_{N}$ and $T_{c}$ become comparable at approximately $0.6 \mathrm{GPa}$. For higher pressures, however, $T_{N}$ reverses the trend and increases in concomitance with the increase of $T_{c}$.

\section{DISCUSSION}

The phase diagram presented in Fig. 3 sets $\mathrm{Sr}_{2} \mathrm{VO}_{3} \mathrm{FeAs}$ apart from other iron-based superconductors. While an increase in $T_{c}$ (e.g., in $\mathrm{LaFeAsO}_{1-x} \mathrm{~F}_{x}$ [43] and $\mathrm{FeSe}$ [44]) as well as an increase (e.g., in $\mathrm{RbEuFe}_{4} \mathrm{As}_{4}$ [45]) and decrease (e.g., in $\left.\mathrm{BaFe}_{2} \mathrm{As}_{2}[46,47]\right)$ in magnetic transition temperatures as a function of pressure have been reported for other materials, the sudden change of trend in $T_{N}$ once $T_{c} \approx$ $T_{N}$ is a novel feature. The simultaneous increase of $T_{N}$ and $T_{c}$ at higher pressures is astonishing as the two orders are usually antagonistic [16-22,48,49]. FeSe is one of the few other examples, where $T_{N}$ and $T_{c}$ increase simultaneously in a certain pressure range $[24,25]$. Please note that, as stated above, for all measured pressures the magnetic fraction of the sample stays close to $100 \%$ at low temperatures while the superconducting volume fraction stays roughly constant, too. Therefore, the magnetic and superconducting order microscopically coexist in a noncompetitive way. This is different from a coexistence at a mesoscopic scale that was reported for other iron-based superconductors (e.g., $\mathrm{CeFeAsO}_{1-x} \mathrm{~F}_{x}$ [50]) and cuprates (e.g., $\mathrm{La}_{1.94} \mathrm{Sr}_{0.06} \mathrm{CuO}_{4}$ [51], $\mathrm{YBa}_{2} \mathrm{Cu}_{3} \mathrm{O}_{6+x}$ [52]).

In the following, we discuss possible origins of the peculiar phase diagram of $\mathrm{Sr}_{2} \mathrm{VO}_{3} \mathrm{Fe}$ As shown in Fig. 3. A trivial explanation is an accidental coincidence of $T_{N}$ and $T_{c}$ at $0.6 \mathrm{GPa}$ 
without a significant coupling of the two orders as reported, e.g., in $\left[\mathrm{Ni}_{0.66} \mathrm{Al}_{0.33}(\mathrm{OH})_{2}\right]\left[\mathrm{TaS}_{2}\right]$ [53]. A so far undetected structural phase transition at $0.6 \mathrm{GPa}$ could be responsible for the sudden change of trend in $T_{N}$. Further experiments are needed to rule out or confirm the existence of a structural transition as a function of pressure.

Another, more intriguing, but up to now hypothetical explanation for the phase diagram of $\mathrm{Sr}_{2} \mathrm{VO}_{3} \mathrm{FeAs}$ is a coupling of the magnetic and superconducting order. Measurements on a second, although less homogeneous, batch of $\mathrm{Sr}_{2} \mathrm{VO}_{3} \mathrm{FeAs}\left(T_{c} \approx 26.5 \mathrm{~K}\right)$ from a different source (see Appendix A) result in a very similar phase diagram. However, the minimum of $T_{N}$ as a function of pressure in this second sample occurs at a slightly higher temperature, which agrees well with the slightly higher $T_{c}$ and might hint at a coupling of the magnetic and superconducting order. In the following, we discuss a possible mechanism for such a coupling based on the electronic properties of the FeAs and $\mathrm{Sr}_{2} \mathrm{VO}_{3}$ subsystems. Band-structure calculations indicate that the hybridization of the $\mathrm{V} 3 d$ state with the Fe $3 d$ state is detrimental to the occurrence of superconductivity [30,31]. In analogy to the Mott transition of $\mathrm{V}_{2} \mathrm{O}_{3}[54,55]$, it is likely that the magnetic transition in the $\mathrm{Sr}_{2} \mathrm{VO}_{3}$ buffer layer is accompanied by a substantial modification of its electronic structure and a localization of the $\mathrm{V} 3 d$ states. With the therefore reduced hybridization of the $\mathrm{V}$ and Fe states, the Fermi surface would become dominated by the Fe $3 d$ bands and exhibit the well-known nesting with a wave vector spanning the hole Fermi surfaces near $\Gamma$ and the electron Fermi surfaces near the $M$ points [56]. A nested Fermi surface is believed to be the key feature promoting the electronic superconducting pairing via spin fluctuations in most Fe-based superconductors [57]. The static magnetism in the vanadium oxide layer (and the likely reorganization of its electronic structure) would therefore be a necessary prerequisite for the occurrence of superconductivity. Evidently, it could be energetically favorable for the system to increase the magnetic transition temperature to gain superconducting condensation energy. Such a mutual coupling could therefore lead to the joint increase of $T_{N}$ and $T_{c}$ above $0.6 \mathrm{GPa}$. The nature of the change in the electronic structure of the $\mathrm{Sr}_{2} \mathrm{VO}_{3}$ layer is, however, not fully clear to date. Magnetic exchange splitting is too small to remove the V $3 d$ bands from the Fermi level [29]. Photoemission spectroscopy [58] and DFT calculations with a GGA+EECE (generalized gradient approximation + exact exchange of correlated electrons) functional [29] indicate that the $\mathrm{V}$ atoms are in a Mott state below the magnetic transition temperature. It is known that $\mathrm{V}_{2} \mathrm{O}_{3}$ exhibits a Mott-Hubbard transition from a paramagnetic metal to an antiferromagnetic insulator with decreasing temperature $[54,55]$. This transition is monotonically suppressed with increasing hydrostatic pressure, which is in accordance with the initial decrease of $T_{N}$ in $\mathrm{Sr}_{2} \mathrm{VO}_{3} \mathrm{Fe} A s$. However, a similar temperature-induced transition of the electronic structure in $\mathrm{Sr}_{2} \mathrm{VO}_{3} \mathrm{FeAs}$, if present, has not been confirmed so far.

\section{CONCLUSION AND OUTLOOK}

In conclusion, we have shown that long-range magnetic order microscopically coexists with superconducting order in
$\mathrm{Sr}_{2} \mathrm{VO}_{3} \mathrm{Fe} A s$. Initially, the application of hydrostatic pressure has opposite effects on the magnetic and superconducting transition temperatures, decreasing the former while increasing the latter until $T_{N} \approx T_{c}$ at $\approx 0.6 \mathrm{GPa}$. For higher pressures, both transition temperatures increase simultaneously, indicating the possibility of a cooperative coupling of the two orders. A possible coupling mechanism via the electronic properties of the FeAs and $\mathrm{Sr}_{2} \mathrm{VO}_{3}$ subsystems has been proposed in this article. Such a speculative cooperative coupling might be interesting for applications since getting control over one order would enable modifications of the other. However, alternative explanations for the phase diagram of $\mathrm{Sr}_{2} \mathrm{VO}_{3} \mathrm{FeAs}$ without a significant coupling, e.g., a pressure-induced structural phase transition, cannot be ruled out, and further experimental and theoretical work is needed.

\section{ACKNOWLEDGMENTS}

This work is partially based on experiments performed at the Swiss Muon Source $\mathrm{S} \mu \mathrm{S}$, Paul Scherrer Institute, Villigen, Switzerland. We gratefully acknowledge the financial support of S.H. by the Swiss National Science Foundation (SNF-Grant No. 200021-159736), of Z.S. by Horizon 2020 [INFRADEV Proposal No. 654000 World class Science and Innovation with Neutrons in Europe 2020(SINE2020)], and of H.O. by JSPS KAKENHI (Grant No. JP16H6439). S.H. thanks C. Wang for useful discussions.

\section{APPENDIX A: COMPARISON WITH SECOND SAMPLE}

The pressure dependence of the magnetic and superconducting transition temperatures was determined by means of muon spin rotation and relaxation $(\mu \mathrm{SR})$ and ac-susceptibility (ACS) measurements for a second sample (denoted sample II) that was synthesized by a different group within our collaboration [42]. Sample II has a lower magnetic volume fraction at low temperatures, and about $10 \%$ of the sample exhibits a transition already at 100-120 K [Fig. 4(a)]. This high-temperature transition could be intrinsic or stem from impurities (e.g., FeAs). All in all, sample I shows a more homogeneous magnetic response with only one magnetic transition with a nearly $100 \%$ volume fraction. Therefore, sample I was chosen to be presented in the main text of this publication. However, the volume sensitive $\mu \mathrm{SR}$ measurements on sample II show that the majority of this sample (about $70 \%$ of the volume) presents the same features as sample I, as becomes evident below.

$5 \mathrm{mT}$ transverse-field (TF) $\mu \mathrm{SR}$ shows that hydrostatic pressure changes the transition temperature $T_{N}$ of the main magnetic transition, but not the low-temperature magnetic volume fraction [Fig. 4(b)], similar to sample I. ACS measurements under pressure were performed with the excitation and pick-up coils wound around the outside of a $\mu$ SR pressure cell [35]. The ACS signal as a function of temperature is shown in the inset of Fig. 4(b) for different pressures. The superconducting onset transition temperature $T_{c}$ was determined via the intersection of two linear approximations of the data above and below the transition, in analogy to the dc-magnetization measurements. $T_{c}$ increases with pressure, but for the highest pressure point the transition is broader and not so well defined. 

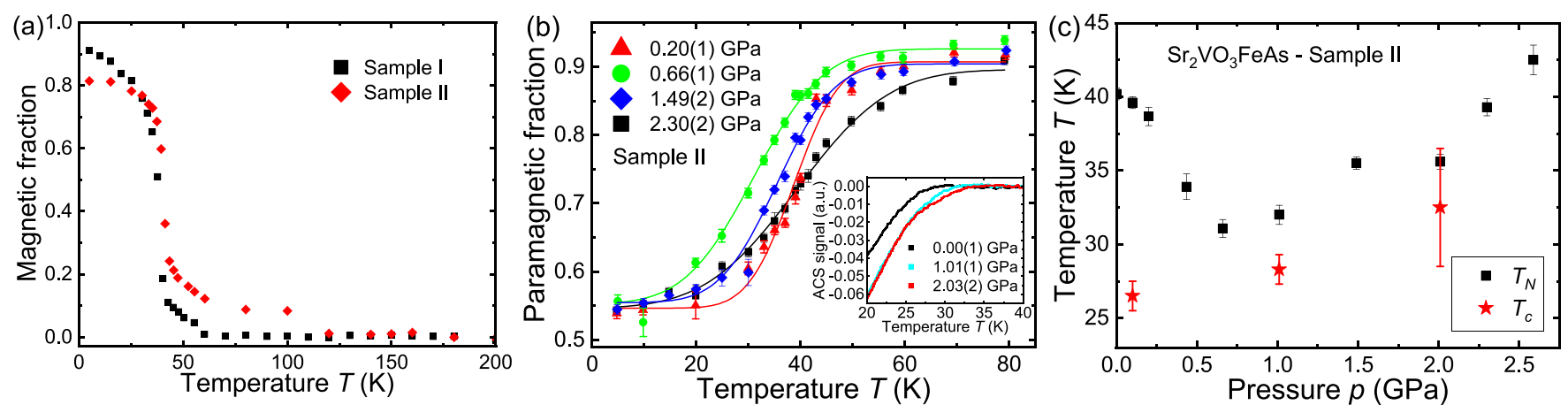

FIG. 4. (a) Temperature dependence of the magnetic volume fraction for $\mathrm{Sr}_{2} \mathrm{VO}_{3} \mathrm{FeAs}$ sample I and sample II at ambient pressure determined by $5 \mathrm{mT}$ TF $\mu$ SR. The error bars are smaller than the data point symbols. A small part of sample II exhibits a transition around $110 \mathrm{~K}$. Further, the magnetic fraction at low temperatures is lower than in sample I. (b) Paramagnetic fraction of sample II as a function of temperature for various pressures. Roughly $50 \%$ of the signal comes from the pressure cell. The solid lines are fits using a normal cumulative distribution function assuming a Gaussian distribution of magnetic transition temperatures. Inset: ACS signal as a function of temperature for various pressures. (c) Temperature-pressure phase diagram of sample II. While the transition temperatures are different from those of sample I, the phase diagrams still shows the same qualitative behavior. $T_{N}$ decreases with pressure until $T_{N} \approx T_{c}$. For higher pressures, $T_{N}$ and $T_{c}$ increase simultaneously.

Figure 4(c) shows the temperature-pressure phase diagram of sample II. $T_{N}$ was determined as the midpoint of a normal cumulative distribution function fit [cf. Fig. 4(b)]. Both $T_{N}$ and $T_{c}$ are in general higher than in sample I. The phase diagrams of the two samples are qualitatively very similar, though. $T_{N}$ initially decreases with pressure until $T_{N} \approx T_{c}$. For higher pressures, $T_{N}$ and $T_{c}$ eventually increase simultaneously. The observed sudden change in the trend of $T_{N}$ once $T_{N} \approx T_{c}$ is therefore not just a feature of a specific batch, but intrinsic to the $\mathrm{Sr}_{2} \mathrm{VO}_{3} \mathrm{FeAs}$ compound.

\section{APPENDIX B: DC-MAGNETIZATION MEASUREMENTS}

The pressure dependence of the superconducting transition temperature $T_{c}$ was determined by dc-magnetization measurements using a commercial superconducting quantum interference device (SQUID) magnetometer. A CuBe anviltype cell with $\mathrm{CuBe}$ gaskets and diamond anvils was used for pressure application in combination with Daphne 7373 oil as a pressure-transmitting medium. Pressure was determined by

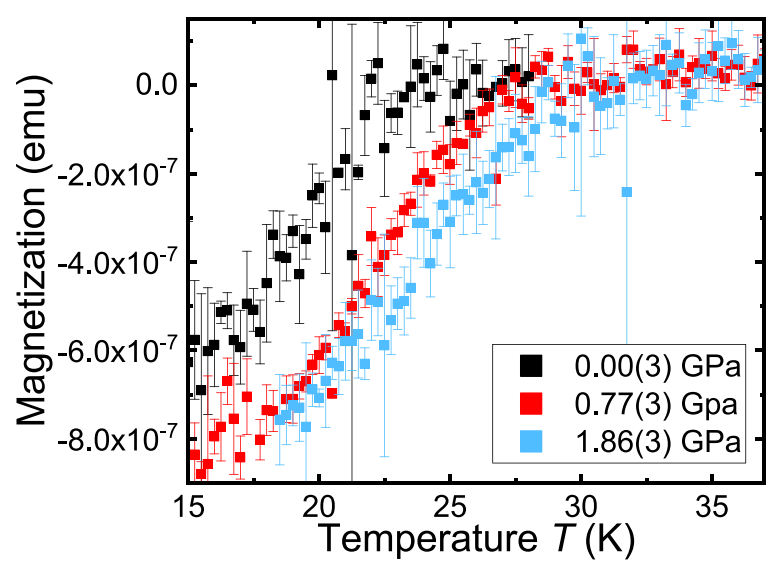

FIG. 5. Magnetization vs temperature for $\mathrm{Sr}_{2} \mathrm{VO}_{3} \mathrm{FeAs}$ at different pressures after subtraction of the cell contribution. The data were shifted to overlap above $T_{c}$ for better comparability. a $\mathrm{Pb}$ manometer [37]. Figure 5 shows representative magnetization data for different pressures. The cell contribution was subtracted and the data were shifted to overlap above $T_{c}$ for better comparability. $T_{c}$ was determined by the intersection of two linear approximations of the data above and below the transition. In agreement with the literature data, $T_{c}$ increases monotonically with pressure [27,42]. Measurements of the superconducting volume fraction are relatively imprecise due to the small sample signal (resulting from the small sample volume). Nonetheless, from the data it is clear that the superconducting volume fraction does not change significantly with pressure.

\section{APPENDIX C: MOESSBAUER SPECTROSCOPY}

${ }^{57} \mathrm{Fe}$ Moessbauer measurements were carried out in an Oxford Instruments He flow cryostat in underpressure mode. The Moessbauer spectrometer consisted mainly of standard WissEl parts. We used a $\mathrm{Rh} / \mathrm{Co}$ source with initial activity of $1.4 \mathrm{GBq}$ and a Si-PIN-detector from KeTek. A high statistics spectrum was taken at room temperature with a larger velocity range. An additional ferrocene absorber was mounted to provide the experimental linewidth at low

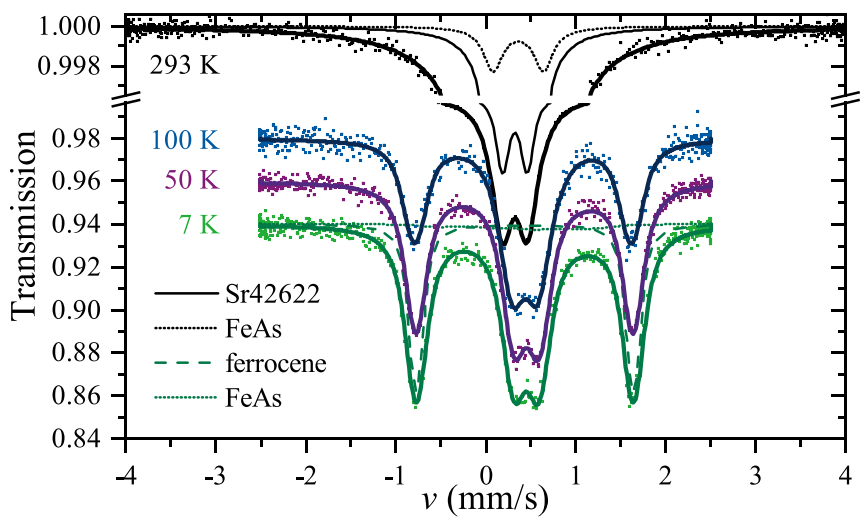

FIG. 6. Moessbauer spectra at different temperatures. 

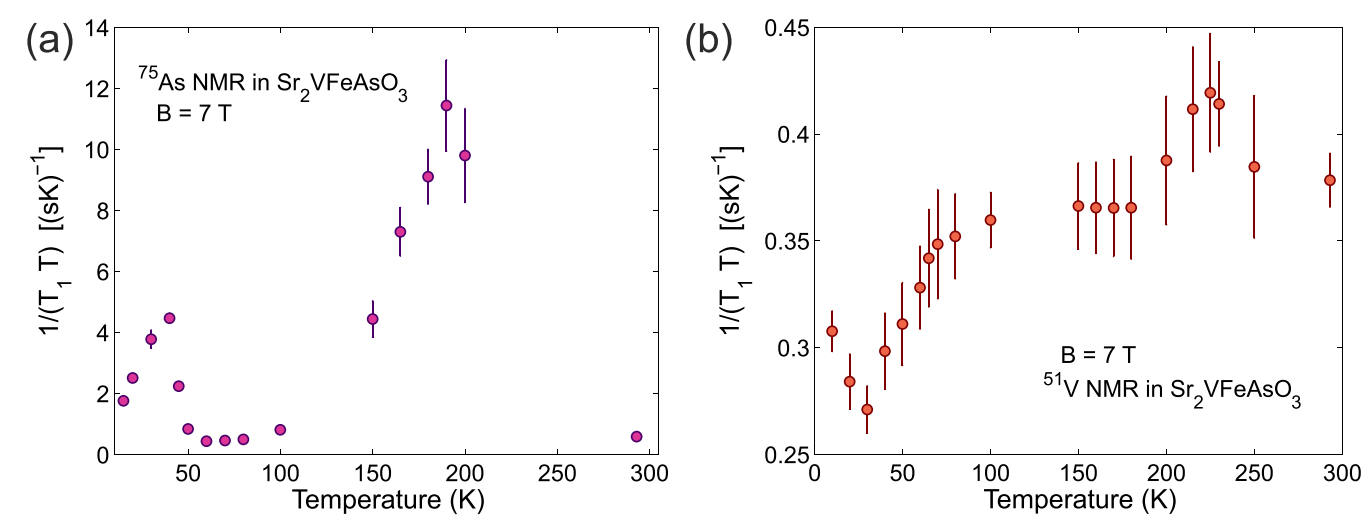

FIG. 7. $1 / T_{1} T$ values as a function of temperature for (a) ${ }^{75} \mathrm{As}-$ and (b) ${ }^{51} \mathrm{~V}-\mathrm{NMR}$ measurement results.

temperatures. All spectra were analyzed in a simultaneous fit using MOESSFIT [59].

The room-temperature Moessbauer spectrum (Fig. 6) consists of a slightly asymmetric $\mathrm{Sr}_{2} \mathrm{VO}_{3} \mathrm{FeAs}$ doublet. The asymmetry can be associated with an FeAs impurity phase, which was included in the fit using the FeAs-model as provided in MOESSFIT [59,60]. All four Moessbauer spectra ( $T=7,50,100,293 \mathrm{~K})$ were fitted simultaneously sharing the same quadrupole splitting of $0.27 \mathrm{~mm} / \mathrm{s}$, which corresponds to $V_{z z}=16.42(4) \mathrm{V} / \AA^{2}$. There is no additional broadening of the doublet or increased hyperfine splitting comparing 7 and $50 \mathrm{~K}$ data. It can be concluded that there is neither static magnetism at the iron atom (upper limit of about $0.1 \mu_{B}$ per $\mathrm{Fe}$ ) nor significant transferred hyperfine fields of iron impurity.

The isomer shift with respect to room-temperature iron is $\delta(T \rightarrow 0)=0.563(2) \mathrm{mm} / \mathrm{s}$. This value is slightly enhanced compared to $0.50(1) \mathrm{mm} / \mathrm{s}$, which is typically measured in Eu122, (Ca,Na)122, and (Na,La) 122 compounds. This can be interpreted as a reduced covalency of the FeAs-bond, or in other words, an increased localization of the $d$-electrons.

\section{APPENDIX D: NUCLEAR MAGNETIC RESONANCE}

${ }^{51} \mathrm{~V}$ and ${ }^{75}$ As nuclear magnetic resonance (NMR) measurements on $\mathrm{Sr}_{2} \mathrm{VO}_{3} \mathrm{FeAs}$ were performed in an applied field of $7.066(1) \mathrm{T}$ and a temperature range from 10 to $293 \mathrm{~K}$.
The NMR line shapes, spin-lattice relaxation time $T_{1}$, and spin-spin relaxation time $T_{2}$ were determined by means of standard spin-echo sequences, with a typical $\pi / 2$ pulse length of $5 \mu \mathrm{s}$ and recycling delays ranging from 0.1 to $30 \mathrm{~s}$. The line shapes were obtained via fast Fourier transform (FFT) of the echo signal, whereas the spin-lattice relaxation times $T_{1}$ were measured via the inversion-recovery method. Considering the selective nature of the applied RF pulses, only the central peak of the spin- $7 / 2{ }^{51} \mathrm{~V}$ nuclei and of the spin- $3 / 2{ }^{75}$ As nuclei was excited. The relevant fit formulas for each case are reported in Ref. [61]. The rather asymmetric positions of the probe nuclei ( $\mathrm{V}$ close to the base of an oxygen pyramid and As at the vertex of an iron pyramid—see, e.g., Ref. [62]) shift and broaden the satellite lines too much to be detectable. In fact, in the ${ }^{75} \mathrm{As}$ case, the quadrupole interaction is so strong that its central NMR line shows second-order broadening effects.

$1 / T_{1} T$ data for ${ }^{75} \mathrm{As}$ [Fig. 7(a)] exhibit peaks around 40 and $200 \mathrm{~K}$, as reported in Refs. [38,41]. A measurement of the ${ }^{75}$ As line in coarse steps (not shown) shows a shift with temperature below $200 \mathrm{~K}$ and a broadening below $100 \mathrm{~K}$, both in agreement with data from the literature [27,41]. 1/ $T_{1} T$ data for ${ }^{51} \mathrm{~V}$ [Fig. 7(b)] exhibit a broad peak around $225 \mathrm{~K}$ and a drop below $\sim 75 \mathrm{~K}$, followed by an upturn below $30 \mathrm{~K}$. The drop below $75 \mathrm{~K}$ is relevant, since it coincides with a peak in the $1 / T_{2}$ dataset for ${ }^{51} \mathrm{~V}$ [Fig. 8(b)] appearing at the same temperature. In contrast to the results reported in Ref. [40],
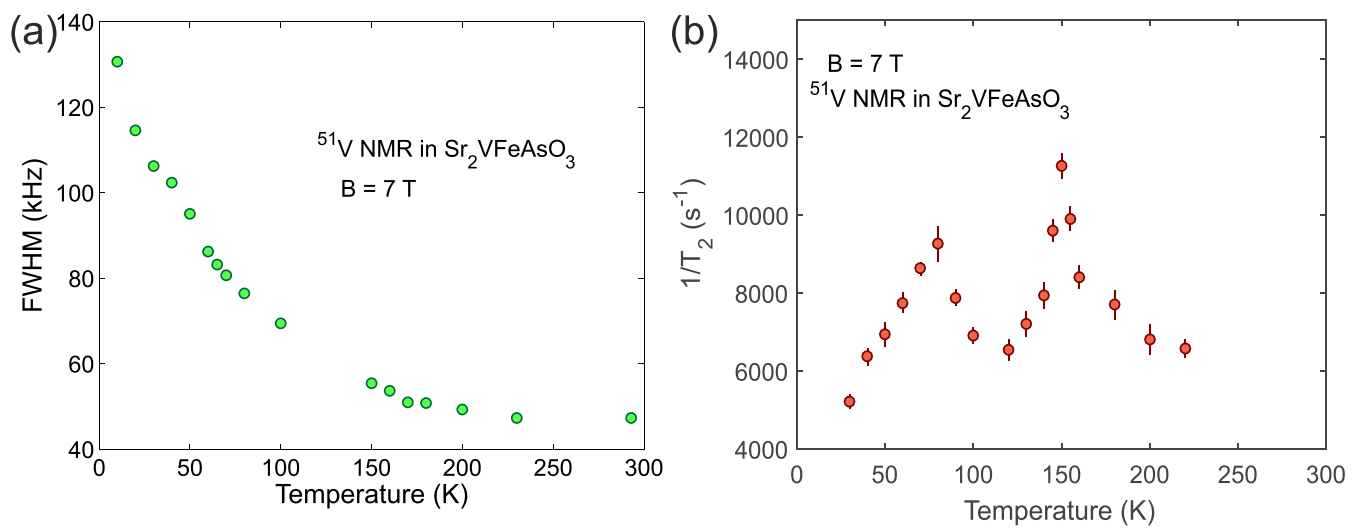

FIG. 8. (a) Full width at half-maximum (FWHM) values of the ${ }^{51} \mathrm{~V}$ line as a function of temperature. (b) ${ }^{51} \mathrm{~V}-1 / T_{2}$ values as a function of temperature. 
$1 / T_{2}$ of ${ }^{51} \mathrm{~V}$ exhibits a second peak around $150 \mathrm{~K}$. The ${ }^{51} \mathrm{~V}$ line barely shifts with temperature, yet it broadens significantly at low $T$ [Fig. 8(a)], in agreement with data reported in Refs. [40,41]. However, no sharp temperature onset is observed for such line broadening.

The shift of the ${ }^{75} \mathrm{As}$ line around $155 \mathrm{~K}$ is believed to stem from a charge- or orbital-type order, with no static magnetism or broken $C_{4}$ symmetry [41]. From the absence of significant shifts in the ${ }^{51} \mathrm{~V}$ line, it was concluded that the $\mathrm{V}$ spins remain disordered down to low temperature, while the peak around $40 \mathrm{~K}$ in the ${ }^{75} \mathrm{As}-1 / T_{1} T$ data was attributed to an ordering of the Fe magnetic moments [41]. Here, however, we argue that the nuclei carrying a magnetic moment might relax too fast (at all temperatures) for the standard NMR setup we used. Due to the inhomogeneity of the sample, the observed signal might stem from a minority of nonmagnetic $\mathrm{V}$ nuclei. Consequently, NMR data cannot exclude an ordering of the vanadium spins. Also, it is not known whether the high magnetic fields required for NMR influence the magnetic properties in a significant way. Indeed, ${ }^{57} \mathrm{Fe}$ Moessbauermeasurement results reported in the literature $[28,32]$ [which, like $\mu$ SR results, are obtained under (nearly) zero-field conditions], as well as our own data (Appendix C), both indicate that the Fe moments do not participate in the static magnetic order. We therefore attribute the static magnetic order to the ordering of the $\mathrm{V}$ magnetic moments.
[1] R. Chevrel, M. Sergent, and J. Prigent, Sur de nouvelles phases sulfurées ternaires du molybdène, J. Solid State Chem. 3, 515 (1971).

[2] B. T. Matthias, H. Suhl, and E. Corenzwit, Ferromagnetic Superconductors, Phys. Rev. Lett. 1, 449 (1958).

[3] W. A. Fertig, D. C. Johnston, L. E. DeLong, R. W. McCallum, M. B. Maple, and B. T. Matthias, Destruction of Superconductivity at the Onset of Long-Range Magnetic Order in the Compound ErRh ${ }_{4} B_{4}$, Phys. Rev. Lett. 38, 987 (1977).

[4] J. W. Lynn, S. Skanthakumar, Q. Huang, S. K. Sinha, Z. Hossain, L. C. Gupta, R. Nagarajan, and C. Godart, Magnetic order and crystal structure in the superconducting $R \mathrm{Ni}_{2} \mathrm{~B}_{2} \mathrm{C}$ materials, Phys. Rev. B 55, 6584 (1997).

[5] C. Bernhard, J. L. Tallon, C. Niedermayer, T. Blasius, A. Golnik, E. Brücher, R. K. Kremer, D. R. Noakes, C. E. Stronach, and E. J. Ansaldo, Coexistence of ferromagnetism and superconductivity in the hybrid ruthenate-cuprate compound $\mathrm{RuSr}_{2} \mathrm{GdCu}_{2} \mathrm{O}_{8}$ studied by muon spin rotation and dc magnetization, Phys. Rev. B 59, 14099 (1999).

[6] J. W. Lynn, B. Keimer, C. Ulrich, C. Bernhard, and J. L. Tallon, Antiferromagnetic ordering of $\mathrm{Ru}$ and $\mathrm{Gd}$ in superconducting $\mathrm{RuSr}_{2} \mathrm{GdCu}_{2} \mathrm{O}_{8}$, Phys. Rev. B 61, R14964 (2000).

[7] G. Cao, S. Xu, Z. Ren, S. Jiang, C. Feng, and Z. Xu, Superconductivity and ferromagnetism in $\operatorname{EuFe}_{2}\left(\mathrm{As}_{1-x} \mathrm{P}_{x}\right)_{2}$, J. Phys.: Condens. Matter 23, 464204 (2011).

[8] S. Nandi, W. T. Jin, Y. Xiao, Y. Su, S. Price, D. K. Shukla, J. Strempfer, H. S. Jeevan, P. Gegenwart, and T. Brückel, Coexistence of superconductivity and ferromagnetism in P-doped $\mathrm{EuFe}_{2} \mathrm{As}_{2}$, Phys. Rev. B 89, 014512 (2014).

[9] S. S. Saxena, P. Agarwal, K. Ahilan, F. M. Grosche, R. K. W. Haselwimmer, M. J. Steiner, E. Pugh, I. R. Walker, S. R. Julian, P. Monthoux, G. G. Lonzarich, A. Huxley, I. Sheikin, D. Braithwaite, and J. Flouquet, Superconductivity on the border of itinerant-electron ferromagnetism in $\mathrm{UGe}_{2}$, Nature 406, 587 (2000).

[10] A. Huxley, I. Sheikin, E. Ressouche, N. Kernavanois, D. Braithwaite, R. Calemczuk, and J. Flouquet, $\mathrm{UGe}_{2}$ : A ferromagnetic spin-triplet superconductor, Phys. Rev. B 63, 144519 (2001).

[11] D. Aoki, A. Huxley, E. Ressouche, D. Braithwaite, J. Flouquet, J.-P. Brison, E. Lhotel, and C. Paulsen, Coexistence of superconductivity and ferromagnetism in URhGe, Nature 413, 613 (2001).
[12] H. Luetkens, H.-H. Klauss, M. Kraken, F. J. Litterst, T. Dellmann, R. Klingeler, C. Hess, R. Khasanov, A. Amato, C. Baines, M. Kosmala, O. J. Schumann, M. Braden, J. HamannBorrero, N. Leps, A. Kondrat, G. Behr, J. Werner, and B. Büchner, The electronic phase diagram of the $\mathrm{LaO}_{1-x} \mathrm{~F}_{x} \mathrm{FeAs}$ superconductor, Nat. Mater. 8, 305 (2009).

[13] J. T. Park, D. S. Inosov, C. Niedermayer, G. L. Sun, D. Haug, N. B. Christensen, R. Dinnebier, A. V. Boris, A. J. Drew, L. Schulz, T. Shapoval, U. Wolff, V. Neu, X. Yang, C. T. Lin, B. Keimer, and V. Hinkov, Electronic Phase Separation in the Slightly Underdoped Iron Pnictide Superconductor $\mathrm{Ba}_{1-x} \mathrm{~K}_{x} \mathrm{Fe}_{2} \mathrm{As}_{2}$, Phys. Rev. Lett. 102, 117006 (2009).

[14] T. Goko, A. A. Aczel, E. Baggio-Saitovitch, S. L. Bud'ko, P. C. Canfield, J. P. Carlo, G. F. Chen, P. Dai, A. C. Hamann, W. Z. Hu, H. Kageyama, G. M. Luke, J. L. Luo, B. Nachumi, N. Ni, D. Reznik, D. R. Sanchez-Candela, A. T. Savici, K. J. Sikes, N. L. Wang, C. R. Wiebe, T. J. Williams, T. Yamamoto, W. Yu, and Y. J. Uemura, Superconducting state coexisting with a phase-separated static magnetic order in $(\mathrm{Ba}, \mathrm{K}) \mathrm{Fe}_{2} \mathrm{As}_{2}$, (Sr,Na)Fe $\mathrm{As}_{2}$, and $\mathrm{CaFe}_{2} \mathrm{As}_{2}$, Phys. Rev. B 80, 024508 (2009).

[15] A. A. Aczel, E. Baggio-Saitovitch, S. L. Budko, P. C. Canfield, J. P. Carlo, G. F. Chen, P. Dai, T. Goko, W. Z. Hu, G. M. Luke, J. L. Luo, N. Ni, D. R. Sanchez-Candela, F. F. Tafti, N. L. Wang, T. J. Williams, W. Yu, and Y. J. Uemura, Muon-spinrelaxation studies of magnetic order and superfluid density in antiferromagnetic $\mathrm{NdFeAsO}, \mathrm{BaFe}_{2} \mathrm{As}_{2}$, and superconducting $\mathrm{Ba}_{1-x} \mathrm{~K}_{x} \mathrm{Fe}_{2} \mathrm{As}_{2}$, Phys. Rev. B 78, 214503 (2008).

[16] N. Ni, M. E. Tillman, J.-Q. Yan, A. Kracher, S. T. Hannahs, S. L. Bud'ko, and P. C. Canfield, Effects of Co substitution on thermodynamic and transport properties and anisotropic $H_{c 2}$ in $\mathrm{Ba}\left(\mathrm{Fe}_{1-x} \mathrm{Co}_{x}\right)_{2} \mathrm{As}_{2}$ single crystals, Phys. Rev. B 78, 214515 (2008).

[17] J.-h. Chu, J. G. Analytis, C. Kucharczyk, and I. R. Fisher, Determination of the phase diagram of the electron-doped superconductor $\mathrm{Ba}\left(\mathrm{Fe}_{1-x} \mathrm{Co}_{x}\right)_{2} \mathrm{As}_{2}$, Phys. Rev. B 79, 014506 (2009).

[18] C. Lester, J.-H. Chu, J. G. Analytis, S. C. Capelli, A. S. Erickson, C. L. Condron, M. F. Toney, I. R. Fisher, and S. M. Hayden, Neutron scattering study of the interplay between structure and magnetism in $\mathrm{Ba}\left(\mathrm{Fe}_{1-x} \mathrm{Co}_{x}\right)_{2} \mathrm{As}_{2}$, Phys. Rev. B 79, 144523 (2009).

[19] C. Bernhard, A. J. Drew, L. Schulz, V. K. Malik, M. Rössle, C. Niedermayer, T. Wolf, G. D. Varma, G. Mu, H.-H. Wen, H. Liu, 
$\mathrm{G}$. $\mathrm{Wu}$, and $\mathrm{X}$. H. Chen, Muon spin rotation study of magnetism and superconductivity in $\mathrm{BaFe}_{2-x} \mathrm{Co}_{x} \mathrm{As}_{2}$ and $\mathrm{Pr}_{1-x} \mathrm{Sr}_{x} \mathrm{FeAsO}$, New J. Phys. 11, 055050 (2009).

[20] E. Wiesenmayer, H. Luetkens, G. Pascua, R. Khasanov, A. Amato, H. Potts, B. Banusch, H.-H. Klauss, and D. Johrendt, Microscopic Coexistence of Superconductivity and Magnetism in $\mathrm{Ba}_{1-x} \mathrm{~K}_{x} \mathrm{Fe}_{2} \mathrm{As}_{2}$, Phys. Rev. Lett. 107, 237001 (2011).

[21] D. K. Pratt, W. Tian, A. Kreyssig, J. L. Zarestky, S. Nandi, N. Ni, S. L. Bud'ko, P. C. Canfield, A. I. Goldman, and R. J. McQueeney, Coexistence of Competing Antiferromagnetic and Superconducting Phases in the Underdoped $\mathrm{Ba}\left(\mathrm{Fe}_{0.953} \mathrm{Co}_{0.047}\right)_{2} \mathrm{As}_{2}$ Compound Using X-Ray and Neutron Scattering Techniques, Phys. Rev. Lett. 103, 087001 (2009).

[22] A. D. Christianson, M. D. Lumsden, S. E. Nagler, G. J. MacDougall, M. A. McGuire, A. S. Sefat, R. Jin, B. C. Sales, and D. Mandrus, Static and Dynamic Magnetism in Underdoped Superconductor $\mathrm{BaFe}_{1.92} \mathrm{Co}_{0.08} \mathrm{As}_{2}$, Phys. Rev. Lett. 103, 087002 (2009).

[23] Y. Laplace, J. Bobroff, F. Rullier-Albenque, D. Colson, and A. Forget, Atomic coexistence of superconductivity and incommensurate magnetic order in the pnictide $\mathrm{Ba}\left(\mathrm{Fe}_{1-x} \mathrm{Co}_{x}\right)_{2} \mathrm{As}_{2}$, Phys. Rev. B 80, 140501(R) (2009).

[24] M. Bendele, A. Amato, K. Conder, M. Elender, H. Keller, H.-H. Klauss, H. Luetkens, E. Pomjakushina, A. Raselli, and R. Khasanov, Pressure Induced Static Magnetic Order in Superconducting FeSe $\mathrm{F}_{1-x}$, Phys. Rev. Lett. 104, 087003 (2010).

[25] M. Bendele, A. Ichsanow, Y. Pashkevich, L. Keller, T. Strässle, A. Gusev, E. Pomjakushina, K. Conder, R. Khasanov, and $\mathrm{H}$. Keller, Coexistence of superconductivity and magnetism in $\mathrm{FeSe}_{1-x}$ under pressure, Phys. Rev. B 85, 064517 (2012).

[26] X. Zhu, F. Han, G. Mu, P. Cheng, B. Shen, B. Zeng, and H.-H. Wen, Transition of stoichiometric $\mathrm{Sr}_{2} \mathrm{VO}_{6} \mathrm{Fe}$ As to a superconducting state at 37.2 K, Phys. Rev. B 79, 220512(R) (2009).

[27] H. Kotegawa, Y. Tao, H. Tou, H. Ogino, S. Horii, K. Kishio, and J.-i. Shimoyama, Pressure dependence of superconducting transition temperature on perovskite-type Fe-based superconductors and NMR study of $\mathrm{Sr}_{2} \mathrm{VFeAsO}_{3}$, J. Phys. Soc. Jpn. 80, 014712 (2011).

[28] J. Munevar, D. R. Sánchez, M. Alzamora, E. Baggio-Saitovitch, J. P. Carlo, T. Goko, A. A. Aczel, T. J. Williams, G. M. Luke, H.-H. Wen, X. Zhu, F. Han, and Y. J. Uemura, Static magnetic order of $\mathrm{Sr}_{4} \mathrm{~A}_{2} \mathrm{O}_{6} \mathrm{Fe}_{2} \mathrm{As}_{2}(\mathrm{~A}=\mathrm{Sc}$ and $\mathrm{V}$ ) revealed by Mössbauer and muon spin relaxation spectroscopies, Phys. Rev. B 84, 024527 (2011).

[29] F. Hummel, Y. Su, A. Senyshyn, and D. Johrendt, Weak magnetism and the Mott state of vanadium in superconducting $\mathrm{Sr}_{2} \mathrm{VO}_{3} \mathrm{FeAs}$, Phys. Rev. B 88, 144517 (2013).

[30] I. R. Shein and A. L. Ivanovskii, Electronic and magnetic properties of superconducting $\mathrm{Sr}_{4} \mathrm{~V}_{2} \mathrm{Fe}_{2} \mathrm{As}_{2} \mathrm{O}_{6}$ versus $\mathrm{Sr}_{4} \mathrm{Sc}_{2} \mathrm{Fe}_{2} \mathrm{As}_{2} \mathrm{O}_{6}$, J. Supercond. Novel Magn. 22, 613 (2009).

[31] K.-W. Lee and W. E. Pickett, $\mathrm{Sr}_{2} \mathrm{VO}_{3} \mathrm{FeAs}$ : A nanolayered bimetallic iron pnictide superconductor, Europhys. Lett. 89, 57008 (2010)

[32] G.-H. Cao, Z. Ma, C. Wang, Y. Sun, J. Bao, S. Jiang, Y. Luo, C. Feng, Y. Zhou, Z. Xie, F. Hu, S. Wei, I. Nowik, I. Felner, L. Zhang, Z. Xu, and F.-C. Zhang, Self-doping effect and successive magnetic transitions in superconducting $\mathrm{Sr}_{2} \mathrm{VFeAsO}_{3}$, Phys. Rev. B 82, 104518 (2010).

[33] M. Tegel, T. Schmid, T. Stürzer, M. Egawa, Y. Su, A. Senyshyn, and D. Johrendt, Possible magnetic order and suppression of superconductivity by $\mathrm{V}$ doping in $\mathrm{Sr}_{2} \mathrm{VO}_{3} \mathrm{Fe} A s$, Phys. Rev. B 82, 140507(R) (2010).

[34] A. Amato, H. Luetkens, K. Sedlak, A. Stoykov, R. Scheuermann, M. Elender, A. Raselli, and D. Graf, The new versatile general purpose surface-muon instrument (GPS) based on silicon photomultipliers for $\mu \mathrm{SR}$ measurements on a continuous-wave beam, Rev. Sci. Instrum. 88, 093301 (2017).

[35] R. Khasanov, Z. Guguchia, A. Maisuradze, D. Andreica, M. Elender, A. Raselli, Z. Shermadini, T. Goko, F. Knecht, E. Morenzoni, and A. Amato, High pressure research using muons at the Paul Scherrer Institute, High Press. Res. 36, 140 (2016).

[36] A. Suter and B. Wojek, Musrfit: A free platform-independent framework for $\mu \mathrm{SR}$ data analysis, Phys. Proc. 30, 69 (2012).

[37] A. Eiling and J. S. Schilling, Pressure and temperature dependence of electrical resistivity of $\mathrm{Pb}$ and $\mathrm{Sn}$ from $1-300 \mathrm{~K}$ and 0-10 GPa - use as continuous resistive pressure monitor accurate over wide temperature range; superconductivity under pressure in $\mathrm{Pb}$, Sn and In, J. Phys. F 11, 623 (1981).

[38] S. Tatematsu, E. Satomi, Y. Kobayashi, and M. Sato, Magnetic ordering in V-Layers of the superconducting system of $\mathrm{Sr}_{2} \mathrm{VFeAsO}_{3}$, J. Phys. Soc. Jpn. 79, 123712 (2010).

[39] A. Yaouanc and P. Dalmas De Réotier, Muon Spin Rotation, Relaxation, and Resonance-Applications to Condensed Matter (Oxford University Press, Oxford, 2011).

[40] K. Ueshima, F. Han, X. Zhu, H.-H. Wen, S. Kawasaki, and G.-q. Zheng, Magnetism and superconductivity in $\mathrm{Sr}_{2} \mathrm{VFeAsO}_{3}$ revealed by ${ }^{75} \mathrm{As}$ - and ${ }^{51} \mathrm{~V}$-NMR under elevated pressures, Phys. Rev. B 89, 184506 (2014).

[41] J. M. Ok, S.-H. Baek, C. Hoch, R. K. Kremer, S. Y. Park, S. Ji, B. Büchner, J.-H. Park, S. I. Hyun, J. H. Shim, Y. Bang, E. G. Moon, I. I. Mazin, and J. S. Kim, Frustration-driven $C_{4}$ symmetric order in a naturally-heterostructured superconductor $\mathrm{Sr}_{2} \mathrm{VO}_{3} \mathrm{FeAs}$, Nat. Commun. 8, 2167 (2017).

[42] H. Kotegawa, T. Kawazoe, H. Tou, K. Murata, H. Ogino, K. Kishio, and J.-i. Shimoyama, Contrasting pressure effects in $\mathrm{Sr}_{2} \mathrm{VFeAsO}_{3}$ and $\mathrm{Sr}_{2} \mathrm{ScFePO}_{3}$, J. Phys. Soc. Jpn. 78, 123707 (2009).

[43] H. Okada, K. Igawa, H. Takahashi, Y. Kamihara, M. Hirano, H. Hosono, K. Matsubayashi, and Y. Uwatoko, Superconductivity under high pressure in LaFeAsO, J. Phys. Soc. Jpn. 77, 113712 (2008).

[44] S. Medvedev, T. M. McQueen, I. A. Troyan, T. Palasyuk, M. I. Eremets, R. J. Cava, S. Naghavi, F. Casper, V. Ksenofontov, G. Wortmann, and C. Felser, Electronic and magnetic phase diagram of $\beta$-Fe1.01Se with superconductivity at $36.7 \mathrm{~K}$ under pressure, Nat. Mater. 8, 630 (2009).

[45] D. E. Jackson, D. VanGennep, W. Bi, D. Zhang, P. Materne, Y. Liu, G.-H. Cao, S. T. Weir, Y. K. Vohra, and J. J. Hamlin, Superconducting and magnetic phase diagram of $\mathrm{RbEuFe}_{4} \mathrm{As}_{4}$ and $\mathrm{CsEuFe}_{4} \mathrm{As}_{4}$ at high pressure, Phys. Rev. B 98, 014518 (2018).

[46] H. Fukazawa, N. Takeshita, T. Yamazaki, K. Kondo, K. Hirayama, Y. Kohori, K. Miyazawa, H. Kito, H. Eisaki, and A. Iyo, Suppression of magnetic order by pressure in $\mathrm{BaFe}_{2} \mathrm{As}_{2}$, J. Phys. Soc. Jpn. 77, 105004 (2008).

[47] A. Mani, N. Ghosh, S. Paulraj, A. Bharathi, and C. S. Sundar, Pressure-induced superconductivity in $\mathrm{BaFe}_{2} \mathrm{As}_{2}$ single crystal, Europhys. Lett. 87, 17004 (2009). 
[48] J. Paglione and R. L. Greene, High-temperature superconductivity in iron-based materials, Nat. Phys. 6, 645 (2010).

[49] H. Hosono, A. Yamamoto, H. Hiramatsu, and Y. Ma, Recent advances in iron-based superconductors toward applications, Mater. Today 21, 278 (2018).

[50] S. Sanna, R. De Renzi, T. Shiroka, G. Lamura, G. Prando, P. Carretta, M. Putti, A. Martinelli, M. R. Cimberle, M. Tropeano, and A. Palenzona, Nanoscopic coexistence of magnetic and superconducting states within the $\mathrm{FeAs}$ layers of $\mathrm{CeFeAsO}_{1-x} \mathrm{~F}_{x}$, Phys. Rev. B 82, 060508(R) (2010).

[51] M.-H. Julien, F. Borsa, P. Carretta, M. Horvatić, C. Berthier, and C. T. Lin, Charge Segregation, Cluster Spin Glass, and Superconductivity in $\mathrm{La}_{1.94} \mathrm{Sr}_{0.06} \mathrm{CuO}_{4}$, Phys. Rev. Lett. 83, 604 (1999).

[52] S. Sanna, G. Allodi, G. Concas, A. D. Hillier, and R. DeRenzi, Nanoscopic Coexistence of Magnetism and Superconductivity in $\mathrm{YBa}_{2} \mathrm{Cu}_{3} \mathrm{O}_{6+x}$ Detected by Muon Spin Rotation, Phys. Rev. Lett. 93, 207001 (2004).

[53] E. Coronado, C. Martí-Gastaldo, E. Navarro-Moratalla, A. Ribera, S. J. Blundell, and P. J. Baker, Coexistence of superconductivity and magnetism by chemical design, Nat. Chem. 2 , 1031 (2010).

[54] D. B. McWhan, A. Menth, J. P. Remeika, W. F. Brinkman, and T. M. Rice, Metal-insulator transitions in pure and doped $\mathrm{V}_{2} \mathrm{O}_{3}$, Phys. Rev. B 7, 1920 (1973).
[55] P. Hansmann, A. Toschi, G. Sangiovanni, T. Saha-Dasgupta, S. Lupi, M. Marsi, and K. Held, Mott-Hubbard transition in $\mathrm{V}_{2} \mathrm{O}_{3}$ revisited, Phys. Status Solidi B 250, 1251 (2013).

[56] I. I. Mazin, $\mathrm{Sr}_{2} \mathrm{VO}_{3} \mathrm{FeAs}$ as compared to other iron-based superconductors, Phys. Rev. B 81, 020507(R) (2010).

[57] A. V. Chubukov, D. V. Efremov, and I. Eremin, Magnetism, superconductivity, and pairing symmetry in iron-based superconductors, Phys. Rev. B 78, 134512 (2008).

[58] T. Qian, N. Xu, Y.-B. Shi, K. Nakayama, P. Richard, T. Kawahara, T. Sato, T. Takahashi, M. Neupane, Y.-M. Xu, X.-P. Wang, G. Xu, X. Dai, Z. Fang, P. Cheng, H.-H. Wen, and H. Ding, Quasinested Fe orbitals versus Mott-insulating V orbitals in superconducting $\mathrm{Sr}_{2} \mathrm{VFeAsO}_{3}$ as seen from angle-resolved photoemission, Phys. Rev. B 83, 140513(R) (2011).

[59] S. Kamusella and H.-H. Klauss, Moessfit, Hyperfine Interact. 237, 82 (2016).

[60] L. Häggström, A. Gustavsson-Seidel, and H. Fjellvåg, A Mössbauer study of helimagnetic FeAs, Europhys. Lett. 9, 87 (1989).

[61] A. Suter, M. Mali, J. Roos, and D. Brinkmann, Mixed magnetic and quadrupolar relaxation in the presence of a dominant static Zeeman Hamiltonian, J. Phys.: Condens. Matter 10, 5977 (1998).

[62] H. Nakamura and M. Machida, Magnetic ordering in blocking layer and highly anisotropic electronic structure of high- $T_{c}$ ironbased superconductor $\mathrm{Sr}_{2} \mathrm{VFeAsO}_{3}$ : LDA $+U$ study, Phys. Rev. B 82, 094503 (2010). 